\title{
Involution matchings, the semigroup of orientation-preserving and orientation-reversing mappings, and inverse covers of the full transformation semigroup
}

\author{
Peter M. Higgins
}

Dept of Mathematical Sciences, University of Essex, Colchester CO4 3SQ email:peteh@essex.ac.uk

AMS Classification Numbers: 20M17, 20M18, 20M19, 20M20.

\begin{abstract}
We continue the study of permutations of a finite regular semigroup that map each element to one of its inverses, providing a complete description in the case of semigroups whose idempotent generated subsemigroup is a union of groups. We show, in two ways, how to construct an involution matching on the semigroup of all transformations which either preserve or reverse orientation of a cycle. Finally, as an application, we use involution matchings to prove that when the base set has at least four members, a finite full transformation semigroup has no cover by inverse subsemigroups that is closed under intersection.
\end{abstract}




\section{Introduction and General Results}

\section{$1.1 \quad$ Background}

In [6] the author introduced the study of permutation matchings, which are permutations on a finite regular semigroup $S$ that map each element to one of its inverses. It follows from Hall's Marriage Lemma that $S$ will possess a permutation matching if and only if $S$ satisfies the condition that $|A| \leq$ $|V(A)|$ for all subsets $A$ of $S$ with set of inverses $V(A)$. Although not all finite regular semigroups have a permutation matching, there are positive results for many important classes. In [7] the author characterised some classes of finite regular semigroups by the nature of their permutation matchings and determined, in terms of Green's relations on principal factors, when a finite orthodox semigroup $S$ has a permutation matching. In this case a permutation matching implies the existence of an involution matching. In Section 1.3 we show how this result may be extended to semigroups whose idempotent-generated subsemigroup is a union of groups.

It is not known whether the semigroup $\mathcal{O}_{n}$ of all order-preserving mappings on a finite $n$-chain has a permutation matching of any kind. It was shown in [6] however that $\mathcal{O P}_{n}$, the semigroup of all orientation-preserving mappings on an $n$-cycle, has a natural involution matching. In Section 2.1 we summarise relevant properties of this semigroup and of $\mathcal{P}_{n}$, the semigroup of all orientation-preserving and orientation-reversing mappings on an $n$-cycle. This latter semigroup, which was introduced in [1] and independently by McAlister in [9], has an intricate structure, which is manifested in the context of the problem of this paper. In Section 3 we construct a dual pair of involution matchings of $\mathcal{P}_{n}$.

There are no known examples of a finite regular semigroup $S$ that has a permutation matching but no involution matching. It was proved in [6] by graph theoretic techniques that $\mathcal{T}_{n}$, the full transformation semigroup on an $n$-set, has a permutation matching but it is not known if $\mathcal{T}_{n}$ has an involution matching. However in Section 4 we show that $\mathcal{T}_{n}(n \geq 4)$ has no involution matching through so-called strong inverses, which allows us to show that $\mathcal{T}_{n}$ $(n \geq 4)$ has no cover by inverse semigroups that is closed under intersection.

Following the texts [8] and [5], we denote the set of idempotents of a semi- 
group $S$ by $E(S)$. We shall write $(a, b) \in V(S)$ if $a$ and $b$ are mutual inverses in $S$ and denote this as $b \in V(a)$ so that $V(a)$ is the set of inverses of $a \in S$. We extend the notation for inverses to sets $A: V(A)=\bigcup_{a \in A} V(a)$; note that this assigns a second meaning to $V(S)$, but the correct interpretation will be clear from context. Standard results on Green's relations, particularly those stemming from Green's Lemma, will be assumed (Chapter 2 of [5], specifically Lemma 2.2.1) and fundamental facts and definitions concerning semigroups that are taken for granted in what follows are all to be found in [5]. We shall sometimes write $\mathcal{G}$ to stand for either of the Green's relations $\mathcal{L}$ or $\mathcal{R}$.

We say that a semigroup $S$ is combinatorial (or aperiodic) if Green's $\mathcal{H}$ relation on $S$ is trivial. A completely 0 -simple combinatorial semigroup is known as a 0-rectangular band. The full transformation semigroup on a base set $X$ is denoted by $\mathcal{T}_{X}$ or by $\mathcal{T}_{n}$ when $X=[n]=\{0,1,2, \cdots, n-1\}$.

Let $C=\left\{A_{i}\right\}_{i \in I}$ be any finite family of finite sets (perhaps with repetition of sets). A set $\tau \subseteq \bigcup A_{i}$ is a transversal of $C$ if there exists a bijection $\phi: \tau \rightarrow C$ such that $t \in \phi(t)$ for all $t \in \tau$. We assume Hall's Marriage Lemma in the form that $C$ has a transversal if and only if Hall's Condition is satisfied, which says that for all $1 \leq k \leq|I|$, the union of any $k$ sets from $C$ has at least $k$ members.

\subsection{Permutation matchings}

Definitions 1.2.1 Let $S$ be any semigroup and let $F=\left\{f \in T_{S}: f(a) \in\right.$ $V(a) \forall a \in S\}$. We call $F$ the set of inverse matchings of $S$. We call $f \in F$ a permutation matching if $f$ is a permutation of $S$; more particularly $f$ is an involution matching if $f^{2}=\varepsilon$, the identity mapping on $S$.

In the remainder of the paper we shall assume that $S$ is regular and finite unless otherwise indicated. We shall often denote a matching simply by ', so that the image of $a$ is $a^{\prime}$. We use the shorthand $a^{\prime \prime}$ as an abbreviation for $\left(a^{\prime}\right)^{\prime}$. We shall work with the family of subsets of $S$ given by $V=\{V(a)\}_{a \in S}$. The members of $V$ may have repeated elements - for example $S$ is a rectangular band if and only if $V(a)=S$ for all $a \in S$. However, we consider the members of $V$ to be marked by the letter $a$, so that $V(a)$ is an unambiguous member of $V$ (strictly, we are using the pairs $\{a, V(a)\},(a \in S)$ ). We summarise 
some results of [6].

Theorem 1.2.2 [6] For a finite regular semigroup $S$ the following are equivalent:

(i) $S$ has a permutation matching;

(ii) $S$ is a transversal of $V=\{V(a)\}_{a \in A}$;

(iii) $|A| \leq|V(A)|$ for all $A \subseteq S$;

(iv) $S$ has a permutation matching that preserves the $\mathcal{H}$-relation; (meaning that $\left.a \mathcal{H} b \Rightarrow a^{\prime} \mathcal{H} b^{\prime}\right)$;

(v) each principal factor $D_{a} \cup\{0\}(a \in S)$ has a permutation matching;

(vi) each 0 -rectangular band $B=\left(D_{a} \cup\{0\}\right) / \mathcal{H}$ has a permutation matching.

In [7, Remark 1.5] it was shown that we may replace 'permutation matching' by 'involution matching' in Theorem 1.2.2 as regards the implications ((i) $\Leftrightarrow(\mathrm{v})) \Leftarrow((\mathrm{iv}) \Leftrightarrow(\mathrm{vi}))$ although the missing forward implication has not been resolved.

\subsection{Permutation matchings for an E-solid semigroup}

Definition 1.3.1 A regular semigroup $S$ is defined to be $E$-solid if $S$ satisfies the condition that for all idempotents $e, f, g \in E(S)$

$$
e \mathcal{L} f \mathcal{R} g \rightarrow \exists h \in E(S): e \mathcal{R} h \mathcal{L} f .
$$

An alternative characterisation of an $E$-solid semigroup is that of a regular semigroup $S$ for which the idempotent-generated subsemigroup $\langle E(S)\rangle$ is a union of groups [3, Theorem 3].

We prove our result on $E$-solid semigroups via the corresponding result for orthodox semigroups. The proof of this latter result involved reducing the general problem to the case of 0-rectangular bands and then showing 
that the corresponding $\mathcal{D}$-class $D$ may be diagonalised in that the $\mathcal{R}$ - and $\mathcal{L}$ classes may be ordered so that all idempotents are contained in rectangular blocks (which then form the maximal rectangular subbands of $D$ ); $S$ then has a permutation matching if and only if, within each $\mathcal{D}$-class of $S$, these blocks are similar in the following sense.

Definition 1.3.2 Let $U_{1}$ and $U_{2}$ be finite rectangular bands, let $m_{i}$ and $n_{i}$ denote the respective number of $\mathcal{R}$-classes and $\mathcal{L}$-classes of $U_{i}(i=1,2)$. We say that $U_{1}$ and $U_{2}$ are similar if $\frac{m_{1}}{n_{1}}=\frac{m_{2}}{n_{2}}$.

Theorem 1.3.3 [7, Theorems 3.7 and 3.1] Let $S$ be a finite orthodox semigroup. Then $S$ has a permutation matching if and only if for each 0-rectangular band $B=\left(D_{a} \cup\{0\}\right) / \mathcal{H}(a \in S)$ the maximal rectangular subbands of $B$ are pairwise similar. In that case the permutation matching of $S$ may be chosen to be an involution matching.

Proposition 1.3.4 Each 0-rectangular band $B=\left(D_{a} \cup\{0\}\right) / \mathcal{H}(a \in S)$ of a finite $E$-solid semigroup $S$ is orthodox.

For completeness, we record a proof of the Proposition but note that the class of all (not necessarily finite) $E$-solid semigroups is a so-called $e$-variety, meaning that the class is closed under the taking of homomorphic images, of direct products, and regular subsemigroups [4]. Also in [4] is shown that a semigroup is orthodox if and only if the same is true of each of its principal factors: (also see [5, Ex. 1.4.13(iv)]).

Proof From the definition of $E$-solidity we see that each principal factor $D_{a} \cup\{0\}$ of $S$ is itself $E$-solid, and $B$ certainly is regular. Next we note that $B$ is $E$-solid through two observations: $H \in E(B)$ if and only if $H=H_{e}$ for some $e \in E(S)$, and $H_{a} \mathcal{G} H_{b}$ in $B$ if and only if $a \mathcal{G} b$ in $S$. Hence if $B$ contain three idempotents $H_{e}, H_{f}$, and $H_{g}$ with $e, f, g \in E(S)$, and they are such that $H_{e} \mathcal{L} H_{f} \mathcal{R} H_{g}$ in $B$, then $e \mathcal{L} f \mathcal{R} g$ in $S$ and by the $E$-solid condition on $S$ we have $e \mathcal{R} h \mathcal{L} g$ for some $h \in E(S)$. We now have $H_{e} \mathcal{R} H_{h} \mathcal{L} H_{f}$ in $B$ and $H_{h} \in E(B)$. Therefore $B$ is $E$-solid.

To show that $B$ is indeed orthodox, first note that by our second observation, $B$ is combinatorial. Then for any two idempotents of $B$, which we now write as $e, f$, we have either that $e f=0 \in E(B)$, or otherwise $e \mathcal{L} g \mathcal{R} f$ for some $g \in E(B)$ whence, since $B$ is $E$-solid, it follows that $h=e f \in E(B)$. 
Therefore $E^{2}(B)=E(B)$, as required.

Theorem 1.3.5 Let $S$ be a finite $E$-solid semigroup. Then $S$ has a permutation matching if and only if the maximal rectangular subbands of each of the 0-rectangular bands $\left(D_{a} \cup\{0\}\right) / \mathcal{H}$ are pairwise similar. Moreover if $S$ has a permutation matching then $S$ has an involution matching.

Proof By Theorem 1.2.2, $S$ has a permutation matching if and only if the same can be said for all $B=\left(D_{a} \cup\{0\}\right) / \mathcal{H}(a \in S)$. By Proposition 1.3.4, each such $B$ is a finite orthodox 0-rectangular band. By Theorem 1.3.3, each such $B$ then has a permutation matching if and only if the maximal rectangular subbands of $B$ are pairwise similar, giving the first statement of Theorem 1.3.5. In this case, again by Theorem 1.3.3, each permutation matching of each $B$ may be chosen to be an involution matching of $B$. Then by (vi) implies (i) in Theorem 1.2.2 as it applies to involutions, we conclude that $S$ itself has an involution matching, thus completing the proof.

\section{Matchings for $\mathcal{O P}$ and $\mathcal{P}_{n}$}

\subsection{The semigroups $\mathcal{O P} \mathcal{P}_{n}$ and $\mathcal{P}_{n}$}

We recap some of the important properties of the semigroups $\mathcal{O P} \mathcal{P}_{n}$ and $\mathcal{P}_{n}$. We also augment these results in order to build a type of calculus for these semigroups. All semigroups under consideration will be subsemigroups of $\mathcal{T}_{n}$. Basic properties of the representation of $\alpha \in \mathcal{T}_{n}$ as a digraph $G(\alpha)$ can be found in the text [5, Section 1.5]. Each component $C$ of $G(\alpha)$ is functional, meaning that each vertex has out-degree 1 so in consequence $C$ consists of a unique cycle $Z(\alpha)$ with a number of directed trees rooted around the vertices of $Z(\alpha)$. The set of cycle points of $G(\alpha)$ are exactly the points in the stable range of $\alpha$, denoted by $\operatorname{stran}(\alpha)$, which are the points of $[n]$ contained in the range of all powers of $\alpha$. Pictures of these digraphs are helpful in seeing what is going on and the reader is invited to draw them where relevant, especially in the examples of Section 4 where they are a natural aid to understanding.

For $\alpha \in \mathcal{T}_{n}$ we write $R=R(\alpha)$ for its range $X \alpha$, while $t=|R(\alpha)|$ will stand for the rank of $\alpha$. The kernel relation of $\alpha$ on $X$ will be denoted as 
$\operatorname{ker}(\alpha)$ with the corresponding partition of $X_{n}$ written as $\operatorname{Ker}(\alpha)$. The set of fixed points of $\alpha$ will be denoted by $F(\alpha)$. Facts from the source paper [1] are listed using the term Result.

Definitions 2.1.1 (i) the cyclic interval $[i, i+t](0 \leq t \leq n-1)$ is the set $\{i, i+1, \cdots, i+t\}$ if $i+t \leq n-1$ and otherwise is the set $\{i, i+1, \cdots, n-1,0,1, \cdots,(i+t)(\bmod n)\}$.

(ii) A finite sequence $A=\left(a_{0}, a_{1}, \cdots, a_{t}\right)$ from $[n]$ is cyclic if there exists no more than one subscript $i$ such that $a_{i}>a_{i+1}$ (taking $t+1=0$ ). We say that $A$ is anti-cyclic if the reverse sequence $A^{r}=\left(a_{t}, a_{t-1}, \cdots, a_{0}\right)$ is cyclic.

Remarks 2.1.2 To say that $A$ is cyclic as in (ii) is equivalent to saying that for some subscript $i, a_{i+1} \leq \cdots \leq a_{t} \leq a_{0} \leq \cdots \leq a_{i}$ and the subscript $i$ with this property is unique unless $A$ is constant. On the other hand $A$ is anti-cyclic means $A^{r}$ is cyclic so that $A$ is anti-cyclic if and only if for some subscript $i$ we have $a_{i+1} \geq \cdots \geq a_{t} \geq a_{0} \geq \cdots \geq a_{i}$ (and $i$ is unique if $A$ is not constant). The properties of cyclicity and anti-cyclicity are inherited by subsequences and by sequences obtained by cyclic re-ordering.

Definition 2.1.3 A mapping $\alpha \in \mathcal{T}_{n}$ is orientation-preserving if its list of images, $(0 \alpha, 1 \alpha, \cdots,(n-1) \alpha)$, is cyclic. The collection of all such mappings is denoted by $\mathcal{O} \mathcal{P}_{n}$. We say that $\alpha \in \mathcal{T}_{n}$ is orientation-reversing if $(0 \alpha, 1 \alpha, \cdots,(n-1) \alpha)$ is anti-cyclic and the collection of all orientationreversing mappings is denoted by $\mathcal{O R}_{n}$.

Result 2.1.4 $\mathcal{O P} \mathcal{P}_{n}$ is a regular submonoid of $\mathcal{T}_{n}$. Each kernel class of $\alpha \in \mathcal{O P} \mathcal{P}_{n}$ is a cyclic interval of $[n]$ and the maximal cycles of the components of the digraph $G(\alpha)$ have the same number of vertices, denoted by $c(\alpha)$.

Definition 2.1.5 Let $\alpha \in \mathcal{O P}{ }_{n}$ be of rank $t \geq 2$. We index the members of $\operatorname{Ker}(\alpha)$ as $K_{i}(0 \leq i \leq t-1)$ in such a way that the set of initial points $a_{i}$ of the cyclic intervals $K_{i}$ satisfy $a_{0}<a_{1}<\cdots<a_{t-1}$, denoting this ordered set by $K(\alpha)$. The list $\left\{K_{0}, K_{1}, \cdots, K_{t-1}\right\}$ is called the canonical listing of the kernel classes of $\alpha$. For $r_{i} \in R(\alpha)$ where $r_{0}<r_{1}<\cdots<r_{t-1}$ we denote the cyclic interval $\left[r_{i}, r_{i}+1, \cdots, r_{i+1}-1\right]$ by $R_{i}$.

Result 2.1.6 ([1], Theorem 3.3) For $t \geq 2$ there is a one-to-one correspondence $\Phi_{0}$ between the set of triples $(K, R, i)$ where $K$ and $R$ are or- 
dered $t$-sets of $X_{n}(0 \leq i \leq t-1)$ and $\left\{\alpha \in \mathcal{O P}_{n}:|X \alpha|=t\right\}$ whereby $(K, R, i) \mapsto \alpha$, where each $a_{j} \in K$ is an initial point of a kernel class of $\alpha$ and $a_{j} \alpha=r_{i+j}(0 \leq i \leq t-1)$, subscripts calculated modulo $t$. Moreover $H_{\alpha}=\left\{\Phi_{0}(K, R, i): i=0,1, \cdots, t-1\right\}$, and so $\left|H_{\alpha}\right|=t$.

Result 2.1.7 (i) The collection $\mathcal{P}_{n}=\mathcal{O P}_{n} \cup \mathcal{O} \mathcal{R}_{n}$ is a regular submonoid of $\mathcal{T}_{n} ; \mathcal{R}$ - $\mathcal{L}$ - and $\mathcal{D}$-classes are determined by equality or kernels, of images, and of ranks respectively (as in $\mathcal{T}_{n}$ and $\mathcal{O P}_{n}$ ).

(ii) the reflection mapping $\gamma:[n] \rightarrow[n]$, whereby $i \mapsto n-i-1(i \in[n])$ is orientation-reversing and $\mathcal{P}_{n}=\langle a, e, \gamma\rangle$, where $a$ is the $n$-cycle $(01 \cdots n-1)$ and $e$ is any idempotent in $\mathcal{O P}_{n}$ of rank $n-1 ;\langle a, e\rangle=\mathcal{O P}{ }_{n}$.

(iii) $\mathcal{O P}_{n} \cap \mathcal{O R}_{n}=\left\{\alpha \in \mathcal{O P}_{n}: \operatorname{rank}(\alpha) \leq 2\right\}$.

(iv) $\left(\mathcal{O} \mathcal{R}_{n}\right)^{2}=\mathcal{O P} \mathcal{P}_{n}, \mathcal{O P}{ }_{n} \cdot \mathcal{O} \mathcal{R}_{n}=\mathcal{O R}_{n} \cdot \mathcal{O P}{ }_{n}=\mathcal{O} \mathcal{R}_{n}$

It is also proved in [1] and in [9] that the respective maximal subgroups of rank $t$ of $\mathcal{O P} \mathcal{P}_{n}$ and of $\mathcal{P}_{n}$ are cyclic groups of order $t$ and dihedral groups of order 2t. Also every non-constant member $\alpha \in \mathcal{O P}_{n}$ factorizes uniquely as $\alpha=a^{r} \phi$ where $a$ is the $n$-cycle as above and $\phi \in \mathcal{O}_{n}$. The constant mappings on $[n]$ comprise $D_{1}$, the lowest $\mathcal{D}$-class of $\mathcal{P}_{n}$. Any permutation of $D_{1}$ is a permutation matching of $D_{1}$ and for that reason $D_{1}$ will not need to feature in our subsequent discussion.

Definition 2.1.8 For $\alpha \in \mathcal{P}_{n}$ we shall write $\rho(\alpha)=(K, R)$, where $K$ and $R$ are the respective sets $K(\alpha)$ of initial points of kernel classes and $R(\alpha)$.

Note that for any $\alpha, \beta \in S=\mathcal{O P}_{n}$ or $\mathcal{P}_{n}, \alpha \mathcal{H} \beta$ if and only if $\rho(\alpha)=\rho(\beta)$. We now extend Result 2.1.6 to $\mathcal{P}_{n}$.

Theorem 2.1.9 For $t \geq 2$ there is a one-to-one correspondence $\Phi$ between the set of quadruples $(K, R, i, k)$ where $K$ and $R$ are ordered $t$-sets of $[n], 0 \leq i \leq t-1, k= \pm 1$ and $\left\{\alpha \in \mathcal{P}_{n}:|X \alpha|=t\right\}$. The correspondence is given by $(K, R, i, k) \mapsto \alpha$, where each $a_{j} \in K$ is an initial point of a kernel class of $\alpha$ and $a_{j} \alpha=r_{i+k j}(0 \leq j \leq t-1)$, subscripts calculated modulo $t$.

Proof The first statement of Result 2.1.6 implies that $\left.\Phi\right|_{k=1}$ maps bijectively onto the set of non-constant mappings in $\mathcal{O P}_{n}$. We show that $\left.\Phi\right|_{k=-1}$ 
maps bijectively onto the set of non-constant mappings in $\mathcal{O R}_{n}$. The equation $K_{j} \alpha=r_{i-j}$ certainly specifies a unique mapping $\alpha=\Phi(K, R, i,-1) \in$ $\mathcal{T}_{n}$, and distinct quadruples yield distinct mappings. We need to check that $\alpha \in \mathcal{O} \mathcal{R}_{n}$. We have however the following equality of two lists:

$$
a_{i+1} \alpha=r_{t-1}>a_{i+2} \alpha=r_{t-2}>\cdots>a_{i} \alpha=r_{0}
$$

It follows from (1) and Remarks 2.1.2 that the image of the cyclic list $K$ under $\alpha$ is anti-cyclic and so $\alpha=\Phi(K, R, i,-1) \in \mathcal{O R}_{n}$; hence $\left.\Phi\right|_{k=-1}$ is a one-to-one mapping into the set of mappings of $\mathcal{O R}_{n}$ of rank at least 2 .

Conversely, let $\alpha \in \mathcal{O} \mathcal{R}_{n}$ be of rank $t \geq 2$. Since multiplication on the right by $\gamma$ defines a bijection of $\mathcal{O P}_{n}$ onto $\mathcal{O R}_{n}$, it follows that the kernel classes of $\alpha$ are cyclic intervals and so $H_{\alpha}$ is determined by a pair of ordered $t$-sets $(K, R)$. Take $i$ such that $a_{i} \alpha=r_{0}$. Then since $a_{i+1}, a_{i+2}, \cdots, a_{i}$ is cyclic and $\alpha \in \mathcal{O} \mathcal{R}_{n}$, it follows that $a_{i+1} \alpha, a_{i+2} \alpha, \cdots, a_{i} \alpha$ is anti-cyclic. However, since $a_{i} \alpha=r_{0}=\min R$, it follows by Remarks 2.1.2 that (1) holds for $\alpha$ and so $\alpha=\Phi(K, R, i,-1)$. Therefore $\left.\Phi\right|_{\mid k=-1}$ is a bijection onto the set of non-constant mappings of $\mathcal{O R}_{n}$. Finally note that for $k= \pm 1$, the rank of $\alpha=\Phi(K, R, i, k)$ is indeed $t=|R|=|K|$.

Corollary 2.1.10 For $t \geq 3$, each $\mathcal{H}$-class $H$ of $\mathcal{P}_{n}$ contained in $D(t)$ is a disjoint union $H=\left(H \cap \mathcal{O} \mathcal{P}_{n}\right) \cup\left(H \cap \mathcal{O R}_{n}\right)$ with each set in the union of cardinal $t$.

Proof Let $H=\left\{\alpha \in \mathcal{P}_{n}: \rho(\alpha)=(K, R)\right\}$. Then by Theorem 2.1.9, $H \cap \mathcal{O P}{ }_{n}=\{\Phi(K, R, i, 1): 0 \leq i \leq t-1\}$ and $H \cap \mathcal{O R}_{n}=\{\Phi(K, R, i,-1):$ $0 \leq i \leq t-1\}$; these two sets each have $t$ members and are disjoint by Result 2.1.7(iii).

We shall refer to the coding of each $\alpha \in \mathcal{P}_{n}$ in the form $(K, R, i, k)$ as the KRik-coordinates of $\alpha$, noting that $(K, R, i, k)=\Phi^{-1}(\alpha)$. We call $i$ and $k$ respectively the shift and the parity of $\alpha$.

Lemma 2.1.11 Let $\alpha \in \mathcal{P}_{n}$ with $\rho(\alpha)=(K, R)$. Then

(i) $\rho(\alpha \gamma)=(K, n-1-R) ; \quad$ (ii) $\rho(\gamma \alpha)=(n-K, R)$;

(iii) $\rho(\gamma \alpha \gamma)=(n-K, n-1-R)$. 
Proof (i) is immediate from definition as is the fact that $R(\gamma \alpha)=R(\alpha)$ in (ii). Continuing in (ii), suppose that $\alpha=\Phi(K, R, i, k)$. Then for $r_{i+k j} \in R$ $(0 \leq j \leq t-1)$, we obtain:

$$
\begin{gathered}
r_{i+k j}(\gamma \alpha)^{-1}=r_{i+k j} \alpha^{-1} \gamma^{-1}=K_{j} \gamma \\
=(n-1)-\left\{a_{j}, a_{j}+1, \cdots, a_{j+1}-1\right\}=\left\{n-a_{j+1}, n-a_{j+1}+1, \cdots, n-a_{j}-1\right\},
\end{gathered}
$$

where $j+1$ is calculated modulo $t$. It follows that $K(\gamma \alpha)=n-K(\alpha)$, thereby establishing (ii). Applying (i) and then (ii) now gives (iii) as follows:

$$
\rho(\gamma \alpha \cdot \gamma)=(K(\gamma \alpha), n-1-R(\gamma \alpha))=(n-K, n-1-R)
$$

Proposition 2.1.12 Let $\alpha=\Phi(K, R, i, k) \in \mathcal{P}_{n}$. Then

(i) $\alpha \gamma=\Phi(K, n-1-R,-(i+1),-k)$;

(ii) if $0 \notin K$ then $\gamma \alpha=\Phi(n-K, R, i-2 k,-k)$;

(iii) if $0 \in K$ then $\gamma \alpha=\Phi(n-K, R, i-k,-k)$;

(iv) if $0 \notin K$ then $\gamma \alpha \gamma=\Phi(n-K, n-1-R, 2 k-(i+1), k)$;

(v) if $0 \in K$ then $\gamma \alpha \gamma=\Phi(n-K, n-1-R, k-(i+1), k)$.

Proof (i) We are working throughout modulo $t$ on subscripts. By Lemma 2.1.11(i) we have $\rho(\alpha \gamma)=(K, n-1-R)$. Now

$$
n-1-R=\left\{n-1-r_{t-1}<n-1-r_{t-2}<\cdots<n-1-r_{0}\right\} .
$$

Let us denote $n-1-r_{-(j+1)}$ by $s_{j}(0 \leq j \leq t-1)$ so that $R(\alpha \gamma)=\left\{s_{0}<\right.$ $\left.s_{1}<\cdots<s_{t-1}\right\}$. Hence $a_{j} \alpha \gamma=r_{i+k j} \gamma=n-1-r_{i+k j}$; now

$$
i+k j=-(-i-k j), \text { so that } a_{j} \alpha \gamma=s_{-(i+1)-k j},
$$

which establishes equation (i).

(ii) By Lemma 2.1.11(ii) we have $\rho(\gamma \alpha)=(n-K, R)$. Since $1 \leq a_{0}$

$$
n-K=n-a_{t-1}<n-a_{t-2}<\cdots<n-a_{0} .
$$


Let us denote $n-a_{-(j+1)}$ by $b_{j}(0 \leq j \leq t-1)$ so that $K(\gamma \alpha)=\left\{b_{0}<b_{1}<\right.$ $\left.\cdots<b_{t-1}\right\}$. Hence:

$b_{j} \gamma \alpha=\left(n-1-\left(n-a_{-(j+1)}\right)\right) \alpha=\left(a_{-(j+1)}-1\right) \alpha=a_{-(j+2)} \alpha=r_{i-k(j+2)}=r_{(i-2 k)-k j}$,

which establishes equation (ii).

(iii) Now since $a_{0}=0$ we have $n-a_{0}=n \equiv 0(\bmod n)$ and so:

$$
n-K=n-a_{0}<n-a_{t-1}<n-a_{t-2}<\cdots<n-a_{1} .
$$

Let us denote $n-a_{-j}$ by $b_{j}(0 \leq j \leq t-1)$ so that $K(\gamma \alpha)=\left\{b_{0}<b_{1}<\right.$ $\left.\cdots<b_{t-1}\right\}$. Hence

$b_{j} \gamma \alpha=\left(n-1-\left(n-a_{t-j}\right)\right) \alpha=\left(a_{-j}-1\right) \alpha=a_{-(j+1)} \alpha=r_{i-k(j+1)}=r_{(i-k)-k j}$,

which establishes equation (iii).

(iv) By Lemma 2.1.11(iii) we have $\rho(\gamma \alpha \gamma)=(n-K, n-1-R)$. Now using (ii) we obtain

$$
b_{j} \gamma \alpha \gamma=r_{(i-2 k)-k j} \gamma=n-1-r_{-(2 k-i+k j)}=s_{(2 k-(i+1))+k j},
$$

which establishes equation (iv).

(v) By Lemma 2.1.11(iii) we have $\rho(\gamma \alpha \gamma)=(n-K, n-1-R)$. Now using (iii) we obtain

$$
b_{j} \gamma \alpha \gamma=r_{i-k-k j} \gamma=n-1-r_{-(k-i+k j)}=s_{(k-(i+1))+k j},
$$

which establishes equation (v).

Example 2.1.13 As an example we find $\gamma \alpha \gamma$ for $\alpha \in \mathcal{O R}_{10}$ given by:

$$
\alpha=\left(\begin{array}{llllllllll}
0 & 1 & 2 & 3 & 4 & 5 & 6 & 7 & 8 & 9 \\
3 & 2 & 2 & 8 & 8 & 6 & 6 & 4 & 3 & 3
\end{array}\right)
$$

so that $n=10, t=5, K=\{1,3,5,7,8\}, R=\{2,3,4,6,8\}$ and $\alpha=$ $\Phi(K, R, 0,-1)$. Since $0 \notin K$, according to Proposition 2.12(iv), we should find that $\gamma \alpha \gamma=\Phi(10-K, 9-R, 2,-1)$ as $i(\gamma \alpha \gamma)=2(-1)-(0+1)=-3 \equiv 2$ $(\bmod 5)$. Now $n-K=\{2,3,5,7,9\}$, and $n-1-R=\{1,3,5,6,7\}$. This accords with the direct calculation of $\gamma \alpha \gamma$, which corresponds to reversing the image line of $\alpha$ (to get $\gamma \alpha$ ) and subtracting the images from $n-1=9$.

$$
\gamma \alpha \gamma=\left(\begin{array}{llllllllll}
0 & 1 & 2 & 3 & 4 & 5 & 6 & 7 & 8 & 9 \\
6 & 6 & 5 & 3 & 3 & 1 & 1 & 7 & 7 & 6
\end{array}\right)
$$




\section{Permutation matchings for $\mathcal{O P} \mathcal{P}_{n}$ and $\mathcal{P}_{n}$}

\subsection{An approach via subset involutions}

Lemma 3.1.1 Let $A \subseteq S$ and let $\left(.^{\prime}\right)$ denote an $\mathcal{H}$-class-preserving involution matching on the set $A$. Then $\left(.^{\prime}\right)$ may be uniquely extended to an involution matching on $A_{\mathcal{H}}=\cup_{a \in A} H_{a}$. In particular, if $A$ meets every $\mathcal{H}$-class of $S$ then $\left(.^{\prime}\right)$ extends uniquely to an $\mathcal{H}$-class-preserving involution of $S$, which we shall call the induced involution matching on $S$.

Proof Since $\left(.^{\prime}\right)$ is $\mathcal{H}$-class-preserving it induces an involution on $A_{\mathcal{H}}$ by $H_{a} \mapsto H_{a^{\prime}}$. We then have an involution matching on $A_{\mathcal{H}}$ defined by $b \mapsto b^{\prime}$ where $b \in H_{a}$ say and $b^{\prime}$ is the unique inverse of $b$ in $H_{a^{\prime}}$.

We now construct what we shall refer to as the natural matching involution for $\mathcal{P}_{n}$, which is the induced involution matching on $\mathcal{P}_{n}$ extending the involution of $\mathcal{O P}_{n}$ recorded in [6].

Theorem 3.1.2 The semigroup $\mathcal{P}_{n}$ has an $\mathcal{H}$-class-preserving involution matching $\left(.^{\prime}\right)$ defined by the rule:

$$
(\alpha=\Phi(K, R, i, k)) \Rightarrow\left(\alpha^{\prime}=\Phi(R, K,-k i, k)\right)
$$

Proof From Theorem 2.1.9 and its proof it follows that (2) defines an $\mathcal{H}$ class-preserving involution that maps each of $\mathcal{O P}$ and $\mathcal{O} \mathcal{R}_{n}$ onto $\mathcal{O P}$ and $\mathcal{O} \mathcal{R}_{n}$ respectively: certainly $\alpha^{\prime \prime}=\alpha$ as $(-k)(-k i)=k^{2} i=i$. It remains only to check that $\left(\alpha, \alpha^{\prime}\right) \in V\left(\mathcal{P}_{n}\right)$ and by symmetry it is enough to verify that $\alpha=\alpha \alpha^{\prime} \alpha$. To this end take $x \in[n]$ with $x \in K_{j}$ say where the kernel classes of $\alpha$ are labelled by subscripts in the canonical order. Then since $-k i+k(i+k j)=-k i+k i+k^{2} j=j$ we obtain:

$$
x \alpha \alpha^{\prime} \alpha=a_{j} \alpha \alpha^{\prime} \alpha=r_{i+k j} \alpha^{\prime} \alpha=a_{-k i+k(i+k j)} \alpha=a_{j} \alpha=x \alpha,
$$

and so $\alpha=\alpha \alpha^{\prime} \alpha$, as required.

Note that for $t=2$ we have $\mathcal{O P}_{n} \cap D_{2}=\mathcal{O R}_{n} \cap D_{2}=D_{2}$ and in this case $k=-k(\bmod t)$. This collapse in the fourth entry of the KRikcoordinates leads to the involution matching taking on the simpler form $\alpha=\Phi(K, R, i) \mapsto \alpha^{\prime}=\Phi(R, K, i)$. 
Examples 3.1.3 Let $n=8, t=4, K=\{0,2,4,6\}, R=\{1,3,5,7\}$ and $\alpha \in \mathcal{O} \mathcal{R}_{8}$ defined by $\alpha=\Phi(K, R, 3,-1)$ so that $\alpha^{\prime}=\Phi(R, K, 3,-1)$.

$$
\alpha=\left(\begin{array}{llllllll}
0 & 1 & 2 & 3 & 4 & 5 & 6 & 7 \\
7 & 7 & 5 & 5 & 3 & 3 & 1 & 1
\end{array}\right) \alpha^{\prime}=\left(\begin{array}{cccccccc}
0 & 1 & 2 & 3 & 4 & 5 & 6 & 7 \\
0 & 6 & 6 & 4 & 4 & 2 & 2 & 0
\end{array}\right) .
$$

For an example in $\mathcal{O P}_{n}$ let us take $n=10, t=6, K=\{0,2,4,7,8,9\}$, $R=\{0,1,2,5,6,7\}$ and $\alpha=\Phi(K, R, 4,1)$. Since $-i=-4=2(\bmod 6)$ we obtain $\alpha^{\prime}=\Phi(R, K, 2,1)$ :

$\alpha=\left(\begin{array}{llllllllll}0 & 1 & 2 & 3 & 4 & 5 & 6 & 7 & 8 & 9 \\ 6 & 6 & 7 & 7 & 0 & 0 & 0 & 1 & 2 & 5\end{array}\right) \alpha^{\prime}=\left(\begin{array}{llllllllll}0 & 1 & 2 & 3 & 4 & 5 & 6 & 7 & 8 & 9 \\ 4 & 7 & 8 & 8 & 8 & 9 & 0 & 2 & 2 & 2\end{array}\right)$.

Example 3.1.4 The natural involution inverse of a group element is not necessarily a group element and nor does the natural involution map $\mathcal{O}_{n}$ into itself. Both features are seen in the following example. Take $n=3, t=2$, $K=\{0,2\}, R=\{1,2\}$, and put $\alpha=\Phi(K, R, 0,1)$ so that $\alpha^{\prime}=\Phi(R, K, 0,1)$ :

$$
\alpha=\left(\begin{array}{lll}
0 & 1 & 2 \\
1 & 1 & 2
\end{array}\right) \quad \alpha^{\prime}=\left(\begin{array}{lll}
0 & 1 & 2 \\
2 & 0 & 2
\end{array}\right)
$$

we see that $\alpha \in E\left(\mathcal{O}_{3}\right) \subseteq E\left(\mathcal{O P}_{3}\right)$ and so is an order-preserving group element while $\alpha^{\prime 2}$ is the constant mapping with range $\{2\}$ and so $\alpha^{\prime}$ is not contained in any subgroup of $\mathcal{O P}_{3}$ and nor is $\alpha^{\prime}$ order-preserving. In the next example $\alpha \in E\left(\mathcal{O}_{2 n}\right)$, but $\alpha^{\prime}$ has no fixed points.

Example 3.1.5 Take $\alpha \in E\left(\mathcal{O}_{2 n}\right)$ so that $R(\alpha)=F(\alpha)$, putting $R(\alpha)$ as the set of odd members of $[2 n]$ and for each even integer $i \in[2 n]$ we put $i \alpha=i+1$. This yields an order-preserving idempotent $\alpha$ on $[2 n]$ of rank $n$ for which

$$
(0 \alpha, 1 \alpha, \cdots,(2 n-2) \alpha,(2 n-1) \alpha)=(1,1,3,3, \cdots, 2 n-1,2 n-1) .
$$

Now $\alpha=\Phi(K, R, 0,1)$ where $K=\{0,2,4, \cdots, 2 n-2\}$ and $R=\{1,3,5, \cdots, 2 n-1\}$. Hence $\alpha^{\prime}=\Phi(R, K, 0,1)$. The kernel classes of $\alpha^{\prime}$ have the form $(i, i+1),(i=1,3, \cdots, 2 n-1)$. We see that $\alpha^{\prime}$ contains the $n$-cycle:

$$
\sigma=(2 n-22 n-42 n-6 \cdots 20),
$$

and for all odd $i$ we have $i \alpha^{\prime}=i-1$. (The digraph $G(\alpha)$ has exactly one component consisting of the $n$-cycle $\sigma$ along with $n$ endpoints, one for each point on $\sigma$.) In particular $c(\alpha)=1$ but $c\left(\alpha^{\prime}\right)=n$. 


\subsection{The dual matching involution of $\mathcal{P}_{n}$}

The mapping on $\mathcal{P}_{n}$ defined by $\alpha \mapsto \alpha \gamma$ (resp. $\alpha \mapsto \gamma \alpha$ ) is an involution on $\mathcal{P}_{n}$ that maps $\mathcal{O P} \mathcal{P}_{n}$ onto $\mathcal{O} \mathcal{R}_{n}$ and maps $\mathcal{O} \mathcal{R}_{n}$ onto $\mathcal{O} \mathcal{P}_{n}$. Additionally, for any $\alpha \gamma \in \mathcal{O R}_{n}\left(\operatorname{resp} . \quad \gamma \alpha \in \mathcal{O R}_{n}\right)$ and $\alpha^{\prime} \in V(\alpha)$ in $\mathcal{O P}_{n}, \gamma \alpha^{\prime} \in V(\alpha \gamma)$ (resp. $\alpha^{\prime} \gamma \in V(\gamma \alpha)$ ) which lies in $\mathcal{O R}_{n}$. The upshot of this is that any permutation matching $\left(.^{\prime}\right)$ on $\mathcal{O} \mathcal{P}_{n}$ may be extended to one on $\mathcal{P}_{n}$ by defining $(\alpha \gamma)^{\prime}=\gamma \alpha^{\prime}$ (or dually, $(\gamma \alpha)^{\prime}=\alpha^{\prime} \gamma$ ). However, if $\left(.^{\prime}\right)$ is an involution matching, the same is not generally true of either of these extensions, even in the case of the natural inverse matching. Lemma 2.1.11 supplies enough information to make this point.

Let $\beta=\Phi(K, R, i,-1) \in \mathcal{O R}_{n}$ so that $\rho(\beta)=(K, R)$. Writing $\beta=\alpha \gamma$ so that $\alpha=\beta \gamma$ we get $\rho(\alpha)=(K, n-1-R)$ and so $\rho\left(\alpha^{\prime}\right)=(n-1-R, K)$. Then for $\bar{\beta}=\gamma \alpha^{\prime}$ we have $\rho(\bar{\beta})=(R+1, K)$. Factorizing $\bar{\beta}$ as $\left(\gamma \alpha^{\prime} \gamma\right) \gamma$ we obtain $\rho\left(\gamma \alpha^{\prime} \gamma\right)=(R+1, n-K-1)$ so that $\rho\left(\left(\gamma \alpha^{\prime} \gamma\right)^{\prime}\right)=(n-K-1, R+1)$. Finally, $\overline{\bar{\beta}}=\gamma\left(\gamma \alpha^{\prime} \gamma\right)^{\prime}$ for which we have $\rho(\overline{\bar{\beta}})=(K+1, R+1)$. In particular we see that in general $\overline{\bar{\beta}} \neq \beta$, as $K+1=K$ if and only if $\beta$ is a member of the group of units of $\mathcal{P}_{n}$. However, by replacing the standard linear ordering by the reverse, or as we shall call it the dual ordering of $[n]$, we automatically obtain a dual involution matching on $\mathcal{P}_{n}$, which we shall denote by $(\overline{)})$. This generates a distinct matching involution of $\mathcal{P}_{n}$ to that of Theorem 3.1.2 and we now seek to express $(\cdot)$ in KRik-coordinates.

Let $\alpha=\Phi(K, R, i, k)$. Under $\left(^{(}\right)$, each $r \in R$ is mapped to the initial point of $r \alpha^{-1}$ in the dual ordering, which is the terminal point $r \alpha^{-1}$ in the standard ordering. It follows that $X \bar{\alpha}=K-1$. Similarly, under $(\bar{*}), R$ becomes the set of initial points of kernel classes in the dual ordering, which is then the set of terminal points of those same classes when expressed in the standard ordering, and so $K(\bar{\alpha})=R+1$. Therefore $\rho(\bar{\alpha})=(R+1, K-1)$. Since the choice of ordering does not affect whether or not $\alpha \in \mathcal{P}_{n}$ preserves or reverses orientation, we may write $\bar{\alpha}=\Phi(R+1, K-1, i(\bar{\alpha}), k)$, where it only remains to determine $i(\bar{\alpha})$.

Theorem 3.2.2 The dual matching involution $\overline{(\cdot)}: \mathcal{P}_{n} \rightarrow \mathcal{P}_{n}$ acts by $\alpha=\Phi(K, R, i, k) \mapsto \Phi(R+1, K-1), i(\bar{\alpha}), k)$ where:

Case (1): $0 \notin K$ and $n-1 \notin R: i(\bar{\alpha})=1+k(1-i)$; 
Case (2): $0 \notin K$ and $n-1 \in R: i(\bar{\alpha})=1-k i$;

Case (3): $0 \in K$ and $n-1 \notin R: i(\bar{\alpha})=k(1-i)$;

Case (4): $0 \in K$ and $n-1 \in R: i(\bar{\alpha})=-k i$.

Moreover, $\alpha$ is in Case (1/4) if and only if $\bar{\alpha}$ is in Case $(1 / 4)$ and $\alpha$ is in Case $(2 / 3)$ if and only if $\bar{\alpha}$ is in Case $(3 / 2)$.

Proof Since $\rho(\bar{\alpha})=(R+1, K-1)$ it follows that $\rho(\alpha \bar{\alpha})=(K, K-1)$. Observe that the unique member of $K-1$ in the kernel class $K_{j}$ is $a_{j+1}-1$ and since $\alpha \bar{\alpha} \in E\left(\mathcal{P}_{n}\right)$ we have:

$$
\begin{gathered}
K_{j} \alpha \bar{\alpha}=a_{j+1}-1 \\
\Rightarrow r_{i+k j} \bar{\alpha}=a_{j+1}-1 \Rightarrow r_{i+j} \bar{\alpha}=a_{k j+1}-1 \\
\Rightarrow r_{j} \bar{\alpha}=a_{k(j-i)+1}-1=a_{(1-k i)+k j}-1 \\
\therefore\left(r_{j}+1\right) \bar{\alpha}=r_{j+1} \bar{\alpha}=a_{(1+k(1-i))+k j}-1
\end{gathered}
$$

The value of $\bar{i}$ now falls into four cases.

Case (1): $0 \notin K$ and $n-1 \notin R$. Since $1 \leq a_{0}$ and $r_{t-1} \leq n-2$ we have, in ascending order:

$$
K-1=\left\{a_{0}-1, a_{1}-1, \cdots, a_{t-1}-1\right\}, R+1=\left\{r_{0}+1, r_{1}+1, \cdots, r_{t-1}+1\right\}
$$

Then putting $j=0$ in (3) yields $\left(r_{0}+1\right) \bar{\alpha}=a_{1+k(1-i)}-1$ and so $\bar{\alpha}=$ $\Phi(R+1, K-1,1+k(1-i), k)$.

Case (2): $0 \notin K$ but $n-1 \in R$ so that the ordered set $R+1=\left\{r_{t-1}+1=\right.$ $\left.0, r_{0}+1, \cdots, r_{t-2}+1\right\}$. Then since $r_{t-1}+1$ is the initial entry of $R+1$ we substitute $j=t-1 \equiv-1(\bmod t)$ into $(3)$ to recover that $\bar{i}$ is $1-k i$ and so $\bar{\alpha}=\Phi(R+1, K-1,1-k i, k)$.

Case (3): $0 \in K$ but $n-1 \notin R$ so that the ordered set $K-1=$ $\left\{a_{1}-1, a_{2}-1, \cdots, a_{t-1}-1, a_{0}-1=n-1\right\}$. As in Case (1) we put $j=0$ in (3) to get $a_{1+k(1-i)}-1$ but since each entry is now listed one place earlier in the ordered set $K-1$ compared to Case (1), we subtract 1 from the outcome in Case (1) to obtain $\bar{\alpha}=\Phi(R+1, K-1, k(1-i), k)$. 
Case (4): $0 \in K$ and $n-1 \in R$ so the ordered set $R+1$ is as in Case (2) and $K-1$ is as in Case (3). Hence $r_{-1}+1$ is the first entry of $R+1$, which, by (3), is mapped to $a_{1-k i}$, which is the entry at position $-k i$ in the list of $K-1$. Hence we obtain $\bar{\alpha}=\Phi(R+1, K-1,-k i, k)$.

Also note that $0 \in K \Leftrightarrow n-1 \in K-1$ and $n-1 \in R \Leftrightarrow 0 \in R+1$. It follows that $\alpha$ is in Case $(1 / 4)$ if and only if $\bar{\alpha}$ is in Case $(1 / 4)$ and that $\alpha$ is in Case $(2 / 3)$ if and only if $\bar{\alpha}$ is in Case (3/2).

Remarks 3.2.3 If we take the union of the natural involution matching $(\cdot)^{\prime}$ on $\mathcal{O} \mathcal{P}_{n}$ with the dual involution matching $\overline{(\cdot)}$ on its complement in $\mathcal{P}_{n}$, we have another involution matching on $\mathcal{P}_{n}$. Since the natural involution matching on $\mathcal{P}_{n}$ is the unique involution matching that extends $(\cdot)^{\prime}$ to $\mathcal{P}_{n}$ while preserving $\mathcal{H}$-classes, it follows that this alternative matching is an example of an involution matching of $\mathcal{P}_{n}$ that does not preserve $\mathcal{H}$.

We may check directly that $\overline{(\cdot)}$ defines an involution: the only nonobvious feature is that the formulas for the shift co-ordinates are self-inverse in Cases (1) and (4) and mutually inverse for Cases (2) and (3) but these are readily checked: for example in Case $(1): 1+k(1-(1+k(1-i)))=i$.

An approach by 'half duals' leads to permutation matchings that are however not involutions. For instance we may look to inverses that map to terminal points of kernel classes while keeping $R$ as the set of initial points of kernel classes of the inverse. In detail, for $\alpha \in \mathcal{P}_{n}$ such that $\rho(\alpha)=(K, R)$ the $\mathcal{H}$-classes defined by the kernel-range pairs $(K, K-1)$ and $(R, R)$ are groups and so there exists a unique inverse $\dot{\alpha}$ of $\alpha$ such that $\rho(\dot{\alpha})=(R, K-1)$. The mapping $\alpha \mapsto \dot{\alpha}$ is then a permutation matching of $\mathcal{P}_{n}$ but not an involution as $\rho(\ddot{\alpha})=(K-1, R-1) \neq \rho(\alpha)$ (unless $\alpha$ lies in the group of units of $\left.\mathcal{P}_{n}\right)$. A dual comment applies to the other half dual where the inverse of $\alpha$ lies in the $\mathcal{H}$-class defined by the pair $(R+1, K)$.

Examples 3.2.4 We take $n=8, t=4, K=\{0,2,4,6\}, R=\{1,3,5,7\}$ and put $\alpha=\Phi(K, R, 0,1)$. We have $R+1=\{0,2,4,6\}=K, K-1=$ $\{1,3,5,7\}=R$. Here we have $0 \in K$ and $n-1=7 \in R$ so that we are in Case (4). By Theorem 3.2.1 we obtain $\bar{\alpha}=\Phi(R+1, K-1,0,1)=\alpha$, and indeed $\alpha$ is an idempotent. In contrast, the natural inverse $\alpha^{\prime}=\Phi(R, K, 0,1)$ :

$\alpha=\alpha^{2}=\left(\begin{array}{llllllll}0 & 1 & 2 & 3 & 4 & 5 & 6 & 7 \\ 1 & 1 & 3 & 3 & 5 & 5 & 7 & 7\end{array}\right)=\bar{\alpha}, \alpha^{\prime}=\left(\begin{array}{llllllll}0 & 1 & 2 & 3 & 4 & 5 & 6 & 7 \\ 6 & 0 & 0 & 2 & 2 & 4 & 4 & 6\end{array}\right)$. 
Next we revisit the first of Examples 3.1.3: $\alpha=\Phi(K, R, 3,-1) \in \mathcal{O R}_{8}$, where $K=\{0,2,4,6\}$ and $R=\{1,3,5,7\}$. Since $0 \in K$ and $n-1=7 \in R$ we are in Case (4) and so $\bar{\alpha}=\Phi(R+1, K-1,3,-1)$ and so $R+1=\{0,2,4,6\}=K$ and $K-1=\{1,3,5,7\}=R$. Hence:

$$
\alpha=\left(\begin{array}{llllllll}
0 & 1 & 2 & 3 & 4 & 5 & 6 & 7 \\
7 & 7 & 5 & 5 & 3 & 3 & 1 & 1
\end{array}\right)=\bar{\alpha} .
$$

Since $\bar{\alpha}=\alpha$, therefore $\overline{\bar{\alpha}}=\alpha$ also, and $\alpha^{3}=\alpha$. In particular, $\bar{\alpha} \neq \alpha^{\prime}$. In general, $\mathcal{O} \mathcal{R}_{n}$ contains no idempotents of rank greater than 2 , so that $\alpha=\alpha^{2}$ is impossible for $\alpha \in \mathcal{O} \mathcal{R}_{n} \backslash \mathcal{O P}{ }_{n}$.

As a third example let $n=10, t=5, K=\{1,3,5,7,8\}, R=\{2,3,4,6,8\}$ with $\alpha=\Phi(K, R, 4,-1)$. Here $0 \notin K$ and $n-1=9 \notin R$ and so we are in Case (1). Note that since $k=-1$ we have for all $i$ that $\bar{i}=1-(1-i)=i$, so in particular $i(\bar{\alpha})=4$ and so $\bar{\alpha}=\Phi(R+1, K-1,4,-1)$. We see that $R+1=\{3,4,5,7,9\}$ and $K-1=\{0,2,4,6,7\}$ :

$\alpha=\left(\begin{array}{llllllllll}0 & 1 & 2 & 3 & 4 & 5 & 6 & 7 & 8 & 9 \\ 2 & 8 & 8 & 6 & 6 & 4 & 4 & 3 & 2 & 2\end{array}\right) \bar{\alpha}=\left(\begin{array}{llllllllll}0 & 1 & 2 & 3 & 4 & 5 & 6 & 7 & 8 & 9 \\ 0 & 0 & 0 & 7 & 6 & 4 & 4 & 2 & 2 & 0\end{array}\right)$.

Beginning with $\bar{\alpha}=\Phi(R+1, K-1,4,-1)$ we have $0 \notin R+1$ and $9 \notin K-1$ so we are (necessarily) again in Case (1). As before $i(\overline{\bar{\alpha}})=4$ and we obtain as expected:

$$
\overline{\bar{\alpha}}=\Phi((K-1)+1,(R+1)-1,4,-1)=\Phi(K, R, 4,-1)=\alpha .
$$

In contrast the natural inverse of $\alpha$ is $\alpha^{\prime}=\Phi(R, K, 1,-1)$.

As an example illustrating Cases $(2 / 3)$ let $n=10, t=6, K=\{1,2,5,7,8,9\}$, $R=\{0,4,5,6,7,9\}$ with $\alpha=\Phi(K, R, 4,1)$. Here $0 \notin K$ but $n-1=9 \in R$, putting $\alpha$ in Case (2). Theorem 3.2.1 gives $i(\bar{\alpha})=1-k i(\alpha)=1-4=-3 \equiv 3$ $(\bmod 6)$. Hence $\bar{\alpha}=\Phi(R+1, K-1,3,1)$, where $0 \in R+1=\{0,1,5,6,7,8\}$ and $9 \notin K-1=\{0,1,4,6,7,8\}$, placing $\bar{\alpha}$ in Case (3). Therefore $\alpha=\left(\begin{array}{llllllllll}0 & 1 & 2 & 3 & 4 & 5 & 6 & 7 & 8 & 9 \\ 6 & 7 & 9 & 9 & 9 & 0 & 0 & 4 & 5 & 6\end{array}\right) \quad \bar{\alpha}=\left(\begin{array}{llllllllll}0 & 1 & 2 & 3 & 4 & 5 & 6 & 7 & 8 & 9 \\ 6 & 7 & 7 & 7 & 7 & 8 & 0 & 1 & 4 & 4\end{array}\right)$, and $\overline{\bar{\alpha}}=\alpha: i(\overline{\bar{\alpha}})=k(1-i(\bar{\alpha}))=1(1-3)=-2 \equiv 4(\bmod 6)=i(\alpha)$. 


\section{Inverse covers and involution matchings for $\mathcal{T}_{n}$}

\subsection{Inverse covers for $\mathcal{T}_{n}$}

In 1971 it was shown by Schein [10] that every finite full transformation semigroup $\mathcal{T}_{n}$ is covered by its inverse subsemigroups, a result that does not extend to the case of an infinite base set, [5, Ex. 6.2.8]. If there existed a cover $\mathcal{A}=\left\{A_{i}\right\}_{1 \leq i \leq m}$ of inverse subsemigroups of $\mathcal{T}_{n}$ with the additional property that the intersection of any pair of semigroups of $\mathcal{A}$ was also an inverse subsemigroup of $\mathcal{T}_{n}$ then we could deduce (as explained below) that $\mathcal{T}_{n}$ had an involution matching. (The semigroups $\mathcal{O} \mathcal{P}_{n}$ and $\mathcal{P}_{n}$ of the previous sections have an inverse cover only if $n \leq 3,[2])$.

It is convenient in what follows to consider the empty set also to be an inverse semigroup. Suppose that $\mathcal{A}=\left\{A_{i}\right\}_{1 \leq i \leq m}$ is an inverse cover of $\mathcal{T}_{n}$ meaning that each $A_{i}$ is an inverse subsemigroup of $\mathcal{T}_{n}$ and that $\cup_{i=1}^{m} A_{i}=\mathcal{T}_{n}$. It follows that, for all $1 \leq i, j \leq m$, the subsemigroup $A_{i, j}=: A_{i} \cap A_{j}$ of $\mathcal{T}_{n}$ has commuting idempotents. Indeed it follows easily from this that $\operatorname{Reg}\left(A_{i, j}\right)$, the set of regular elements of $A_{i, j}$, forms an inverse subsemigroup of $A_{i, j}$. However it does not automatically follow that $A_{i, j}=\operatorname{Reg}\left(A_{i, j}\right)$.

Let $S$ be an arbitrary semigroup and $a \in S$. We say that $b \in V(a)$ is a strong inverse of $a$ if the subsemigroup $\langle a, b\rangle$ of $S$ is an inverse semigroup. We denote the set of strong inverses of $a$ by $S(a)$. We next observe that $S$ has an inverse cover if and only if every element of $S$ has a strong inverse for, on the one hand, if every element $a$ has a strong inverse then $S$ is covered by its inverse subsemigroups $\langle a, b\rangle$ where $b \in S(a)$. On the other hand suppose that $S$ has an inverse cover. Take $a \in S$ and choose an inverse subsemigroup $A_{a}$ of $S$ containing $a$ and let $b$ be the (unique) inverse of $a$ in $A_{a}$. Then $A=\langle a, b\rangle$ is a subsemigroup of $A_{a}$ with commuting idempotents and every element of $A$ is regular as for any product $p=c_{1} c_{2} \cdots c_{k} \in A\left(c_{i} \in\{a, b\}\right)$ we see that $p^{\prime}=c_{k}^{\prime} c_{k-1}^{\prime} \cdots c_{1}^{\prime}$ is an inverse of $p$ in $A$, where we take $a^{\prime}=b$ and $b^{\prime}=a$, because both products take place within the inverse semigroup $A_{a}$. It follows that to prove that a given semigroup $S$ has an inverse cover is equivalent to showing that $S(a)$ is non-empty for every $a \in S$.

The following general observation applies to any inverse cover $\mathcal{A}=\left\{A_{i}\right\}_{i \in I}$ of an arbitrary semigroup $S$ : if the pairwise intersection of any two mem- 
bers of $\mathcal{A}$ is an inverse subsemigroup of $S$ then the same is true of arbitrary intersections. To see this let $J \subseteq I$ and consider $A=\cap_{j \in J} A_{j}$. Either $A$ is the empty inverse subsemigroup or we may choose $a \in A$ and consider an arbitrary $A_{j}(j \in J)$. Then $a$ has a unique inverse $a^{-1}$ in $A_{j}$. Now let $k \in J$. By hypothesis, $A_{j} \cap A_{k}$ is an inverse subsemigroup of $S$ that contains a. Since $A_{j} \cap A_{k}$ is an inverse subsemigroup of the inverse semigroup $A_{j}$, it follows that the unique inverse of $a$ in $A_{j} \cap A_{k}$ is $a^{-1}$. Since $k \in J$ was arbitrary, it follows that $a^{-1} \in A$ and so $A$ is indeed an inverse subsemigroup of $S$. We shall say that $S$ has a closed inverse cover if $S$ has a cover by inverse subsemigroups for which all pairwise intersections of its members are themselves inverse semigroups.

Theorem 4.1.1 For a finite semigroup $S$ :

(i) if $S$ has a closed inverse cover then $S$ has an involution matching by strong inverses.

(ii) If every element $a \in S$ has a unique strong inverse $b$ then $S$ has a closed inverse cover

$$
\mathcal{C}=\left\{\cap_{i=1}^{k} U_{i}, U_{i}=\langle a, b\rangle, a \in S, k \geq 1\right\} .
$$

(iii) If $a$ is a group element of $S$ then $a^{-1}$, the group inverse of $a$ in $S$, is the unique strong inverse of $a$.

Proof (i) Suppose there exists a closed inverse cover $\mathcal{A}=\left\{A_{0}\right\}_{0 \leq i \leq m}$ of $S$ where, without loss, we include $\emptyset$ as $A_{0}$. The collection $\mathcal{A}$ is partially ordered by inclusion. Since every partial order may be extended to a total order, we may order the members of $\mathcal{A}$ in such a way that if $A_{i} \subset A_{j}$, then $A_{i}$ appears before $A_{j}$ in the list. This is assumed in the following argument.

We now show how $\mathcal{A}$ could be used to build an involution matching $\left({ }^{\prime}\right)$ of $S$ for which $a^{\prime} \in S(a)$. First $A_{0}$ has an involution matching $\left(.^{\prime}\right)$ in the empty function. Next let $U=\cup_{i=0}^{k} A_{i}(k \geq 1)$. Suppose inductively that we have extended the involution $\left(\cdot^{\prime}\right)$ to $V=\cup_{i=0}^{k-1} A_{i}$ and that for each $a \in A_{j}$, $(0 \leq j \leq k-1) a^{\prime} \in A_{j}$ (so that $a^{\prime} \in S(a)$ ). Let $a \in A_{k}$. Suppose first that $a \in V$ so that $a \in A_{j}$ for some $0 \leq j \leq k-1$. Then $a^{\prime}$ is already defined and by the nature of the linear order we have imposed on $\mathcal{A}, A_{i}=A_{j} \cap A_{k} \subseteq V$, with $i \leq j \leq k-1$. Therefore by induction we have $a^{\prime} \in A_{j} \cap A_{k}$ and so the 
induction continues in the case where $a \in A_{k} \cap V$.

Otherwise $a \notin V$. Then there exists a unique strong inverse $a^{\prime} \in S(a) \cap$ $A_{k}$. What is more $a^{\prime} \notin V$ for if to the contrary $a^{\prime} \in A_{j}$ say $(0 \leq j \leq k-1)$ then $a^{\prime}$ is again a member of the inverse semigroup $A_{i}=A_{j} \cap A_{k} \subseteq V$, where $i \leq k-1$. In this event, $\left(a^{\prime}\right)^{\prime}$ is already defined and would be an inverse of $a^{\prime}$ in $A_{j} \cap A_{k}$, whence $\left(a^{\prime}\right)^{\prime}=a$. But then $a \in A_{j}$, contrary to our choice of $a$. It follows that $a^{\prime} \notin V$ and so we may extend the involution by strong inverses $\left(\cdot^{\prime}\right)$ to $U=V \cup A_{k}$ by setting $a^{\prime}$ as the unique inverse of $a$ in $A_{k} \backslash V$ for all $a \in A_{k} \backslash V$. Therefore we see that in both cases the induction continues. Since $\mathcal{A}$ covers $S$, the process terminates when $k=m$, yielding an involution matching by strong inverses $\left(.^{\prime}\right)$ of $S$.

(ii) Let $U, V \in \mathcal{A}$ and suppose that $U \cap V \neq \emptyset$. For any $a \in U \cap V$ let $b$ be the unique strong inverse of $a$. Let $u \in V(a)$ in the inverse semigroup $U$. Then $u \in S(a)$, whence $u=b$. We may draw the corresponding conclusion for the inverse $v \in V(a) \cap V$, so that $u=b=v$. In particular $b \in U \cap V$, whence it follows that $U \cap V$ is an inverse subsemigroup. Therefore by adjoining all intersections $U_{1} \cap U_{2} \cap \cdots \cap U_{k}(k \geq 2)$ of members $U_{i} \in \mathcal{A}$ to the inverse cover $\mathcal{A}$ we generate a closed inverse cover for $S$.

(iii) Clearly $a^{-1} \in S(a)$. Consider an arbitrary $b \in S(a)$ and let $e=a b$, $f=b a$. Then we have $e \mathcal{R} a \mathcal{L} f$ in $S$. Then since $H_{a}$ is a group we have $f e \mathcal{H} b$. Since $b \in S(a)$ it follows that $e f=f e \in E(S)$. But then $a \mathcal{H} e f=f e \mathcal{H} b$ and so $b, a^{-1} \in V(a)$ with $b \mathcal{H} a^{-1}$, whence $b=a^{-1}$ by uniqueness of inverses within an $\mathcal{H}$-class.

Remark 4.1.2 As observed prior to Theorem 4.1.1, for any semigroup with a closed inverse cover, the intersection of any collection of members of $\mathcal{A}$ is also an inverse subsemigroup of $S$. This allows the argument of the previous proof to be extended to arbitrary semigroups through the Axiom of choice and transfinite induction (the part (iii) argument makes no use of finiteness). Part (ii) of Theorem 4.1.1 is a partial converse of part (i). It remains open as to whether or not the full converse holds.

In the next section, we shall prove that in general $\mathcal{T}_{n}$ has no involution matching by strong inverses, from which it follows from the contrapositive of Theorem 4.1.1(i) that $\mathcal{T}_{n}$ has no closed inverse cover. 


\subsection{Closed inverse covers for $\mathcal{T}_{n}$ do not exist}

In this section our context throughout will be $\mathcal{T}_{n}$. The account here of the construction of strong inverses in $\mathcal{T}_{n}$ follows [5, Section 6.2].

Let $\alpha \in \mathcal{T}_{n}$. For $x \in X_{n}$ the depth of $x$, denoted by $d(x)$, is the length of the longest dipath in $G(\alpha)$ ending at $x$; if $x \in \operatorname{stran}(\alpha)$ we conventionally define $d(x)=\infty$. Note that $d(x)=k<\infty$ if and only if $x \in X \alpha^{k} \backslash X \alpha^{k+1}$. The height of $x$, denoted by $h(x)$ is the least positive integer $k$ such that $d\left(x \alpha^{k}\right) \geq d(x)+k+1$; again we take $h(x)=\infty$ if $x \in \operatorname{stran}(\alpha)$. The height of $x$ is the length of the dipath which begins at $x$ and terminates at the first point $u$ which is also the terminal point of some dipath that is longer than the dipath from $x$ to $u$. A necessary condition for membership of $S(a)$ is the following.

Lemma 4.2.1 Let $\beta \in S(\alpha)$. Then for all $x \in X \alpha, x \beta$ is a member of $x \alpha^{-1}$ of maximal depth.

When constructing strong inverses, the correct treatment of the endpoints of $G(\alpha)$, which are those $x \in X_{n}$ for which $d(x)=0$, is more complicated. The next parameter is defined on the vertices of $G(\alpha)$ in terms of some fixed but arbitrary $\beta \in \mathcal{T}_{n}$, but is only significant when $\beta \in V(\alpha)$. For each $x \in X_{n}$ the grasp $g(x)$ of $\alpha$ is the greatest non-negative integer $k$ such that $x \alpha^{k} \beta^{k}=x$.

Lemma 4.2.2 Let $\beta \in S(\alpha)$. If $d(x)=0$, and $h(x)=h$ then $x \beta=y$ satisfies $g(y) \geq g(x)+1$ and $y \alpha^{h+1}=x \alpha^{h}$.

Lemmas 4.2.1 and 4.2.2 are all we require here. However, if $\beta \in \mathcal{T}_{n}$ satisfies these conditions together with the equality $x \beta \alpha \cdot \alpha^{g(x)+1} \beta^{g(x)+1}=$ $x \alpha^{g(x)+1} \beta^{g(x)+1} \cdot \beta \alpha$, it may then be proved that that $\beta \in S(\alpha)$. We may show from this point that $\mathcal{T}_{n}$ has an inverse cover for the lemmas represent the two stages in the construction of a particular type of strong inverse $\beta \in S(\alpha)$ : Lemma 4.2.1 applies to points of positive depth in $G(\alpha)$, while for each endpoint $x$ we may follow the dipath (of length $k$ say) from $x$ until we meet a point $u$ of depth exceeding $k$. Then $u \beta^{t}$ has already been defined for all $0 \leq t \leq k+1$ and we then put $x \beta=x \alpha^{k} \beta^{k+1}$. This stage can always be carried out and indeed this $\beta \in S(\alpha)$ is uniquely determined by the choices made in determining $X \alpha \beta$. The outcome of this is a particular strong inverse 
$\beta \in S(\alpha)$ for such a $\beta$ will also satisfy the additional condition and indeed the set of all idempotents $\alpha^{t} \beta^{t}, \beta^{s} \alpha^{s}$ then commute with each other, a necessary and sufficient condition for $\langle\alpha, \beta\rangle$ to be an inverse semigroup.

The main result of this section is the following.

Theorem 4.2.3 The full transformation semigroup $\mathcal{T}_{n}$ has a closed inverse cover if and only if $n \leq 3$.

Lemma 4.2.4 For $n \leq 3, \mathcal{T}_{n}$ has a closed inverse cover.

Proof For $n=1,2$ we note that $\mathcal{T}_{n}$ is a union of groups so the claim follows from Theorem 4.1.1. Although $\mathcal{T}_{3}$ is not a union of groups, we may verify that each $\alpha \in \mathcal{T}_{3}$ has a unique strong inverse as follows.

In general, an element $\alpha \in \mathcal{T}_{n}$ is a group element if and only if $X \alpha=X \alpha^{2}$. It follows that all members of $\mathcal{T}_{3}$ of ranks 1 or 3 are group elements. There are $3^{3}-3-3 !=18$ mappings in $\mathcal{T}_{3}$ of rank 2 . All of those with two components are idempotent (these number $3 \times 2=6$ ). Those with one component for which $\left|X \alpha^{2}\right|=2$ are group elements (these also number 6). This leaves $18-6-6=6$ mappings of rank 2 with a single component and for which $\left|X \alpha^{2}\right|=1$. These are evidently the 6 mappings $\alpha$ of the form $a \mapsto b \mapsto c \mapsto c$ where $\{a, b, c\}=\{1,2,3\}$, which we denote for our current purposes by $(a b c)$. Observe that each such $\alpha$ has a unique strong inverse, which is $\alpha^{\prime}=(b a c)$. The result now follows by Theorem 4.1.1(ii).

Examples 4.2.5 For $\alpha=(123)$ we have $S(\alpha)=\alpha^{\prime}=(213), \alpha \alpha^{\prime}$ : $1 \mapsto 1,2,3 \mapsto 3, \alpha^{\prime} \alpha: 1,3 \mapsto 3,2 \mapsto 2$. The inverse subsemigroup $U_{3}=$ $\left\langle\alpha, \alpha^{\prime}\right\rangle$ is a 5 -element combinatorial Brandt semigroup with zero element given by $\alpha^{2}=\alpha^{\prime 2}$, which is the constant mapping with range $\{3\}$. The subsemigroups $U_{1}, U_{2}, U_{3}$ are pairwise disjoint. However not all intersections of distinct members of the inverse cover $\mathcal{C}=\{\langle a, b\rangle: b \in S(a)\}$ are empty: for example consider the mapping $\gamma: 1 \mapsto 3,2,3 \mapsto 1$, which is its own strong inverse. Since $\gamma^{2}=\alpha \alpha^{\prime}$ we obtain $\langle\gamma\rangle \cap\left\langle\alpha, \alpha^{\prime}\right\rangle=\left\{\alpha \alpha^{\prime}\right\}$.

By way of contrast, let us examine a subsemigroup $\left\langle\alpha, \alpha_{1}\right\rangle$, where $\alpha_{1} \in$ $V(\alpha) \backslash S(\alpha)$ : we take $\alpha_{1}=(321)$. Then $e=\alpha \alpha_{1} \in E\left(\mathcal{T}_{3}\right)$ and has fixed point set of $\{1,2\}$ with $3 e=2$. Also $f=\alpha^{2} \in E\left(\mathcal{T}_{3}\right)$ is the constant mapping with range $\{3\}$, so that $e f=f$. However $f e$ is the constant mapping onto 2 
and so idempotents do not commute in $U=\left\langle\alpha, \alpha_{1}\right\rangle$. In fact $U$ is a 7-element regular subsemigroup of $S$, containing all three constant mappings, as $\alpha_{1}^{2}$ is the constant with range $\{1\}$.

Given Lemma 4.2.4 we now need to prove that $\mathcal{T}_{n}$ does not have a closed inverse cover for $n \geq 4$. The remaining substantial task is to show that $\mathcal{T}_{4}$ has no involution matching through strong inverses for that implies that $\mathcal{T}_{4}$ has no closed inverse cover. For $n \geq 5$ we then consider the copy of $\mathcal{T}_{4}$ embedded in $\mathcal{T}_{n}$ defined by $T=\left\{\alpha \in \mathcal{T}_{n}: k \alpha=k \forall k \geq 5\right\}$. Suppose that $\mathcal{C}$ were a closed inverse cover for $\mathcal{T}_{n}$. If $\alpha \in T$ then for any $\beta \in S(\alpha)$ we have $\beta \in T$. It follows that $\mathcal{C}_{T}=\{A \cap T: A \in \mathcal{C}\}$ would be a closed inverse cover for $T$, which is isomorphic to $\mathcal{T}_{4}$, which would then also have such a cover. Therefore, to complete the proof of our theorem, it remains only to show that $\mathcal{T}_{4}$ does not have an involution matching by strong inverses.

First we identify every member of $\mathcal{T}_{4}$ that possesses a unique strong inverse, a collection that includes all mappings of ranks 1 or 4 as these are group elements. Indeed for any rank we only need consider non-group elements, which are the mappings $\alpha$ such that $X \alpha^{2}$ is proper subset of $X \alpha$.

Consider mappings of rank 3. It follows that $\left|X \alpha^{2}\right| \leq 2$. If $\alpha$ has two components, since $X \alpha^{2} \neq X \alpha$, it follows that $\alpha$ has an isolated fixed point $d$ say and a second component of the form $a \mapsto b \mapsto c \mapsto c$, which has a unique strong inverse $b \mapsto a \mapsto c \mapsto c, d \mapsto d$. If $\alpha$ has just one component with $|\operatorname{stran}(\alpha)|=2$ then $\alpha$ necessarily now has the form $a \mapsto b \mapsto c \mapsto d \mapsto c$, which has a unique strong inverse, which is the mapping $b \mapsto a \mapsto d \mapsto c \mapsto d$. Otherwise $|\operatorname{stran}(\alpha)|=1$ and $\alpha$ has the form $a \mapsto b \mapsto c \mapsto d \mapsto d$, which has a unique strong inverse given by $c \mapsto b \mapsto a \mapsto d \mapsto d$. We conclude that all mappings of ranks 1,3 , or 4 each have a unique strong inverse.

Finally consider mappings of rank 2 . Since we may assume there exists a point $x \in X \alpha \backslash \operatorname{stran}(\alpha)$ (as otherwise $\alpha$ is a group element) it follows that we are restricted to mappings $\alpha$ with a single component and that component has a fixed point. The two remaining cases are:

$$
\text { A: the form of a ' } \mathrm{Y} \text { ': } \alpha=\left(\begin{array}{llll}
a & b & c & d \\
c & c & d & d
\end{array}\right) \text { or B: the form } \beta=\left(\begin{array}{llll}
a & b & c & d \\
b & d & d & d
\end{array}\right) \text {. }
$$

We next act the strong inverse operator $S(\cdot)$ for mappings of these two 
types. We will see that this generates a set of four 9-cycles, with each mapping within a cycle sharing the same fixed point. Consequently these cycles are pairwise disjoint.

The given mapping $\alpha$ of type A has exactly two strong inverses, both of which are of type B:

$$
\beta_{1}:\left(\begin{array}{llll}
a & b & c & d \\
d & d & a & d
\end{array}\right) \beta_{2}=\left(\begin{array}{llll}
a & b & c & d \\
d & d & b & d
\end{array}\right)(B)
$$

The mapping $\beta$ of type $\mathrm{B}$ also has exactly two strong inverses, the first of type $\mathrm{A}$, the second of type $\mathrm{B}$ :

$$
\alpha_{1}:\left(\begin{array}{llll}
a & b & c & d \\
d & a & a & d
\end{array}\right)(A) \beta_{2}:\left(\begin{array}{llll}
a & b & c & d \\
d & a & d & d
\end{array}\right)(B)
$$

Consider the collection $C$ of all mappings of rank 2 with two strong inverses and a common fixed point, $d$. There are 3 mappings of type A and 6 of type $\mathrm{B}$, so that $C$ has 9 members. We use the symbols $\alpha$ and $\beta$, with appropriate subscripts, to denote mappings of types $\mathrm{A}$ and of $\mathrm{B}$ respectively.

The strong inverse operator $S(\cdot)$ acting on a point in $C$ outputs exactly two distinct mappings, which are also members of $C$, in accord with the rules (5) and (6). Let us write $\alpha_{1}=\alpha$ for the type A mapping above. We write $\beta_{1} \rightarrow \alpha_{1} \rightarrow \beta_{2}$ with the arrow indicating the first map is a strong inverse of the second (so that the reverse arrow is equally valid). We now act the operator $S(\cdot)$ on the rightmost member of our sequence, which will produce as outputs the previous member and a new sequence member. Bearing in mind rules (5) and (6) our sequence $C$ will thus take on the form:

$$
C: \beta_{1} \rightarrow \alpha_{1} \rightarrow \beta_{2} \rightarrow \beta_{3} \rightarrow \alpha_{2} \rightarrow \beta_{4} \rightarrow \beta_{5} \rightarrow \alpha_{3} \rightarrow \beta_{6} \cdots
$$

The output of $S(\gamma)$ when acting on $\gamma \in C$ comprises two distinct mappings, neither of which is $\gamma$, and one of which is the predecessor of $\gamma$ in the sequence. Eventually the output $S(\gamma)$ will produce a repeated member of $C$ (in addition to the predecessor of $\gamma$ ), which must appear at least two steps before $\gamma$. However, all such members of $C$, apart from $\beta_{1}$, have already had their two strong inverses appear in $C$, and so cannot have $\gamma$ as a third strong inverse. Therefore the repeated sequence member is necessarily $\beta_{1}$. Hence $C$ is a cycle of length $l$ say with $l \geq 2$ and $l \mid 9$, and so $l=3$ or $l=9$. However $l=3$ would imply that $\beta_{1}$ and $\beta_{2}$ were mutual inverses, which is not the case. Therefore $l=9$ and and the cycle $C$ is completed by $\beta_{6} \rightarrow \beta_{1}$. 
Suppose now that $\mathcal{T}_{4}$ possessed an involution $\left(.^{\prime}\right)$ by strong inverses. Any mapping $\alpha$ with a unique strong inverse $\beta$ is necessarily paired with $\beta$ under '. This includes all mappings in $\mathcal{T}_{4}$ except for the mappings which are the vertices of the four disjoint 9-cycles we have just identified. Each member of such a 9 -cycle $C$ is then paired with an adjacent partner in that cycle, but since 9 is odd, this is not possible and so we have a contradiction. Therefore $\mathcal{T}_{4}$ has no involution by strong inverses, which implies by Theorem 4.1.1(i) that $\mathcal{T}_{4}$ has no inverse cover closed under the taking of intersections.

Remarks 4.2.6 We may explicitly calculate the 9-cycle $C$ that contains the mapping $\alpha$ above, denoted here as $\alpha_{1}$, through repeated use of rules (5) and (6) as follows. We write $S\left(\alpha_{1}\right)=\left\{\beta_{1}, \beta_{2}\right\}$, with the $\beta_{i}$ as given in (5). Then following (7) the subsequent members of $C$ are $\beta_{2} \rightarrow \beta_{3}=$ $\left(\begin{array}{llll}a & b & c & d \\ d & c & d & d\end{array}\right) \rightarrow \alpha_{2}=\left(\begin{array}{llll}a & b & c & d \\ b & d & b & d\end{array}\right) \rightarrow \beta_{4}=\left(\begin{array}{cccc}a & b & c & d \\ d & a & d & d\end{array}\right) \rightarrow \beta_{5}=$ $\left(\begin{array}{llll}a & b & c & d \\ b & d & d & d\end{array}\right) \rightarrow \alpha_{3}=\left(\begin{array}{llll}a & b & c & d \\ d & a & a & d\end{array}\right) \rightarrow \beta_{6}=\left(\begin{array}{llll}a & b & c & d \\ c & d & d & d\end{array}\right) \rightarrow \beta_{7}=\beta_{1}=$ $\left(\begin{array}{llll}a & b & c & d \\ d & d & a & d\end{array}\right)$, giving the anticipated 9-cycle $C$.

Running down the ranks from 4 to 1 , elementary combinatorial considerations give that:

$$
\left|E\left(\mathcal{T}_{4}\right)\right|=1+2\left(\begin{array}{l}
4 \\
2
\end{array}\right)+\left(3\left(\begin{array}{l}
4 \\
1
\end{array}\right)+(2)(2)\left(\begin{array}{l}
4 \\
2
\end{array}\right)\right)+4=1+12+36+4=53 .
$$

In a similar fashion, bracketing term sum contributions from a common rank, the number of non-idempotent self-inverse elements is given by:

$$
\left(\left(\begin{array}{l}
4 \\
2
\end{array}\right)+\frac{1}{2}\left(\begin{array}{l}
4 \\
2
\end{array}\right)\right)+(2)(3)\left(\begin{array}{l}
4 \\
2
\end{array}\right)+2\left(\begin{array}{l}
4 \\
2
\end{array}\right)=9+36+12=57
$$

The number of mappings with a distinct unique strong inverse is given by:

$$
\left.\left.\left(3 !+2 !\left(\begin{array}{l}
4 \\
3
\end{array}\right)\right)+\left((3)(2 !)\left(\begin{array}{l}
4 \\
3
\end{array}\right)+(2)(2)\left(\begin{array}{l}
4 \\
2
\end{array}\right)\right)+4 !\right)+(2)(2)\left(\begin{array}{l}
4 \\
2
\end{array}\right)\right)=14+96=110 .
$$

The number of mappings with exactly two strong inverses is $4 \times 9=36$, giving the total of $(53+57)+110+36=256=4^{4}=\left|\mathcal{T}_{4}\right|$. The graph of strong inverses of $\mathcal{T}_{4}$ then consists of 110 singletons, 55 pairs, and four 9-cycles. In particular the above analysis shows that $\mathcal{T}_{4}$ does possess a permutation 
matching by strong inverses. We may use one of these permutations to construct an involution matching of $\mathcal{T}_{4}$. (There are $2^{4}=16$ such permutations, determined by the 2 choices of orientation of the 4 cycles). First consider the 9 -cycle explicitly calculated above in which all mappings fix a point $d$. The mapping $\alpha=\alpha_{1}$ has an idempotent inverse $\varepsilon_{d}=\left(\begin{array}{llll}a & b & c & d \\ b & b & b & d\end{array}\right)$. We then remove the pair $\left(\varepsilon_{d}, \varepsilon_{d}\right)$ from our permutation, replacing it by $\left(\varepsilon_{d}, \alpha\right)$ and pair up the remaining 8 members of the associated 9-cycle in neighbouring pairs. We repeat this procedure with the other three cycles, noting that there is no repetition of idempotents used in our pairings. This then yields an involution matching for $\mathcal{T}_{4}$.

We close with an example showing however that in general $\mathcal{T}_{n}$ does not possess a permutation matching by strong inverses.

Example 4.2.7 Consider the following pair of members of $\mathcal{T}_{8}$ :

$$
\alpha_{1}=\left(\begin{array}{cccccccc}
1 & 2 & 3 & 4 & 5 & 6 & 7 & 8 \\
2 & 3 & 4 & 5 & 5 & 3 & 8 & 4
\end{array}\right) \alpha_{2}=\left(\begin{array}{cccccccc}
1 & 2 & 3 & 4 & 5 & 6 & 7 & 8 \\
2 & 3 & 4 & 5 & 5 & 8 & 3 & 4
\end{array}\right) .
$$

The two mappings are identical except for the interchange of the images of 6 and 7 , and so their digraphs are isomorphic. They share a common range: $X \alpha_{1}=X \alpha_{2}=\{2,3,4,5,8\}$. Moreover for each $x \in X \alpha_{i}(i=1,2)$ there is a unique member of $y \in x \alpha_{i}^{-1}$ of maximal depth and so by Lemma 4.2.1 we see that any strong inverse $\beta_{i} \in S\left(\alpha_{i}\right)$ has the following form:

$$
\beta_{1}=\left(\begin{array}{cccccccc}
1 & 2 & 3 & 4 & 5 & 6 & 7 & 8 \\
- & 1 & 2 & 3 & 5 & - & - & 7
\end{array}\right) \beta_{2}=\left(\begin{array}{cccccccc}
1 & 2 & 3 & 4 & 5 & 6 & 7 & 8 \\
- & 1 & 2 & 3 & 5 & - & - & 6
\end{array}\right) .
$$

In each case the points of depth zero are 1,6, and 7. For both mappings and for any strong inverses $\beta_{i}$ we see that $g(1)=3$ so that for any choice of $y=$ $1 \beta_{i}$ we have by Lemma 4.2 .2 that $g(y) \geq 4$, which implies that $y=1 \beta_{i}=5$. To determine $6 \beta_{1}$ we note that $6 \alpha_{1}=3$ and so $d\left(6 \alpha_{1}\right)=2>0+1=d(6)+1$; hence $h(6)=1$ and $g(6)=0$. Writing $y=6 \beta_{1}$ we have by Lemma 2.2 that $g(y) \geq 1$ and $y \alpha_{1}^{2}=6 \alpha_{1}=3$ so that $y=6 \beta_{1}=1$. By the same argument with 6 replaced by 7 we obtain $7 \beta_{2}=1$.

Finally consider $7 \beta_{1}$. We have $7 \alpha_{1}=8$ so we see that $d\left(7 \alpha_{1}\right)=1$ and $g(7)=1, h(7)=2$ as $d\left(7 \alpha_{1}^{2}\right)=d(4)=3>2+0=2+d(7)$ while $d(7 \alpha)=d(8)=1 \ngtr 1+0$. Hence we have by Lemma 4.2 .2 that $y=7 \beta_{1}$ must satisfy $g(y) \geq 2$ and $y \alpha^{3}=7 \alpha^{2}=4$ so that $7 \beta_{1}=1$. By symmetry we 
also obtain $6 \beta_{2}=1$. Therefore each of the $\alpha_{i}$ has a unique strong inverse $\beta_{i}$ :

$$
\beta_{1}=\left(\begin{array}{llllllll}
1 & 2 & 3 & 4 & 5 & 6 & 7 & 8 \\
5 & 1 & 2 & 3 & 5 & 1 & 1 & 7
\end{array}\right) \quad \beta_{2}=\left(\begin{array}{llllllll}
1 & 2 & 3 & 4 & 5 & 6 & 7 & 8 \\
5 & 1 & 2 & 3 & 5 & 1 & 1 & 6
\end{array}\right) .
$$

We now consider a third mapping $\beta \in \mathcal{T}_{8}$ and a putative strong inverse $\beta^{\prime} \in S(\beta)$. As before we have $X \beta=\{2,3,4,5,8\}$ and again Lemma 4.2.2 gives the following unique partial definition of $\beta^{\prime}$ :

$$
\beta=\left(\begin{array}{cccccccc}
1 & 2 & 3 & 4 & 5 & 6 & 7 & 8 \\
2 & 3 & 4 & 5 & 5 & 8 & 8 & 4
\end{array}\right) \quad \beta^{\prime}=\left(\begin{array}{cccccccc}
1 & 2 & 3 & 4 & 5 & 6 & 7 & 8 \\
- & 1 & 2 & 3 & 5 & - & - & -
\end{array}\right) .
$$

We see that $8 \beta^{\prime} \in\{6,7\}$; for the moment let us make the choice $8 \beta^{\prime}=7$ and henceforth denote $\beta^{\prime}$ by $\beta_{1}^{\prime}$. The points of zero depth are again 1,6 , and 7 and the same analysis that applied to the $\alpha_{i}$ again yields $1 \beta_{1}^{\prime}=5$. Next we note that $g(6)=0$ and $h(6)=2$ as $d\left(6 \alpha^{2}\right)=d(4)=3>2+0$ but $d(6 \alpha)=d(8)=1 \ngtr 1+0$. Hence $y=6 \beta_{1}^{\prime}$ must satisfy $g(y) \geq 1$ and $y \beta^{3}=6 \beta^{2}=4$ so that $y=6 \beta_{1}^{\prime}=1$. Finally we have $g(7)=1$ and $h(7)=2$ as for $h(6)$. Hence $y=7 \beta^{\prime}$ must satisfy $g(y) \geq 2$ and $y \beta^{3}=6 \beta^{2}=4$ so that $y=7 \beta^{\prime}=1$ also. We have then identified one strong inverse of $\beta_{1}^{\prime} \in S(\beta)$. Similarly there exists a second strong inverse $\beta_{2}^{\prime} \in S(\beta)$ determined by the alternative choice $8 \beta_{2}^{\prime}=6$. Exchanging the roles of the symbols 6 and 7 makes no difference to the images of the other domain points in that we again obtain that $1 \beta_{2}^{\prime}=5,6 \beta_{2}^{\prime}=7 \beta_{2}^{\prime}=1$. Therefore we find that

$\beta_{1}^{\prime}=\beta_{1}=\left(\begin{array}{cccccccc}1 & 2 & 3 & 4 & 5 & 6 & 7 & 8 \\ 5 & 1 & 2 & 3 & 5 & 1 & 1 & 7\end{array}\right) \quad \beta_{2}^{\prime}=\beta_{2}=\left(\begin{array}{llllllll}1 & 2 & 3 & 4 & 5 & 6 & 7 & 8 \\ 5 & 1 & 2 & 3 & 5 & 1 & 1 & 6\end{array}\right)$.

The upshot of all this is that we have a set of three members of $\mathcal{T}_{8}$ in $U=$ $\left\{\alpha_{1}, \alpha_{2}, \beta\right\}$ such that the set $S(U)$ of all strong inverses of elements of $U$ is the two-element set $S(U)=\left\{\beta_{1}, \beta_{2}\right\}$. It follows that there is no permutation matching $(\cdot)^{\prime}$ on $\mathcal{T}_{8}$ that maps the set $U$ into the set $S(U)$, thereby yielding the result mentioned earlier, which we now formally state.

Corollary 4.2.8 There is no permutation matching $\left(.^{\prime}\right)$ of $\mathcal{T}_{n}(n \geq 8)$ such that, for all $a \in \mathcal{T}_{n}, a^{\prime}$ is a strong inverse of $a$.

Proof Example 4.2.7 shows the corollary is true for $n=8$. For $n \geq 9$ we may extend the above example with each of the the mappings $\alpha_{1}, \alpha_{2}, \beta$ acting identically on all integers exceeding 8 . Since any strong inverse preserves components, we again obtain the conclusion that $S\left(\alpha_{1}\right)=\left\{\beta_{1}\right\}, S\left(\alpha_{2}\right)=$ $\left\{\beta_{2}\right\}$ and $S(\beta)=\left\{\beta_{1}, \beta_{2}\right\}$, which implies the result. 


\section{References}

[1] Catarino P.M. and Higgins P.M., The monoid of orientation-preserving mappings on a chain, Semigroup Forum Vol. 58 (1999), 190-206.

[2] Catarino P.M. Higgins P.M., Levi I., On inverse subsemigroups of the semigroup of orientation-preserving or orientation-reversing mappings, Algebra and Discrete Mathematics, 19, (2015) No. 2, 162-171.

[3] Hall, T.E., On regular semigroups, J. of Algebra, 24, 1-24 (1973).

[4] Hall, T.E., Identities for existence varieties of regular semigroups, Bull. Austral. Math. Soc. 40 (1989), 59-77.

[5] Higgins, P.M., Techniques of semigroup theory, OUP (1992).

[6] Higgins, P.M., Permutations of a semigroup that map to inverses, Semigroup Forum, (2014) 89: 169 - 182.

[7] Higgins, P.M., Orthodox semigroups and permutation matchings, Semigroup Forum, (2017) 94: 167-175.

[8] Howie, J.M., Fundamentals of semigroup theory, Clarendon Press (1995).

[9] McAlister, D.B., Semigroups generated by a group and an idempotent, Comm. in Alg. (1998) 26(2), 243-254.

[10] Schein, B.M, A symmetric semigroup of transformations is covered by its inverse subsemigroups, Acta. Mat. Acad. Sci. Hung. 22, (1971), 163-71. 